\title{
Adaptive Real-time HEVC Encoding of Emergency Scenery Video
}

\author{
A. S. Panayides and A. G. Constantinides \\ Dept. of Electrical and Electronic Engineering \\ Imperial College London \\ a.panagidis@imperial.ac.uk
}

\author{
M. S. Pattichis \\ Dept. of Electrical and Computer Engineering \\ University of New Mexico \\ Albuquerque, USA
}

\author{
E. Kyriacou \\ Dept. of Computer Science and Engineering \\ Frederick University \\ Cyprus
}

C. S. Pattichis

Dept. of Computer Science

University of Cyprus

Cyprus

\begin{abstract}
This paper proposes the use of an adaptive HEVC encoding framework for the wireless transmission of real-time emergency scenery video. The goal of the proposed approach is to adapt HEVC encoding methods and parameters so as to meet time-varying constraints on available bandwidth, video quality, and performance. We present an example in real-time video communications by requiring that the encoding rate can meet or exceed the source video frame-rate at a reasonable delay.
\end{abstract}

Emergency video, adaptive encoding, encoding, wireless transmission, quality assessment

\section{INTRODUCTION \& METHODOLOGY}

Real-time wireless communication of emergency scenery video aims at efficient disaster crisis management. In emergency situations, it can accommodate effective assessment of the disaster incident. Linked with appropriate telemedicine systems (such as AvarisNet [1]), it facilitates on-site coordination of rescue teams, triage, and ambulance dispatch of the most serious injuries to the nearest hospital.

In terms of wireless video communication, the challenge is to provide the appropriate level of video quality that will deliver an accurate reconstruction of the emergency incident to the management site, in real-time [2]. Moreover, given the unpredictable nature of the disaster, the system has to adapt to the available bandwidth given the best available wireless infrastructure. Based on the afore-described opposing constraints, the goal is to maximize video quality without exceeding constraints on available bandwidth, while allowing for real-time encoding (e.g., see [3]).

\section{Prelimenary Results \& Discussion}

An emergency scenery video of a simulated earthquake response, as a part of the Cyprus Emergency Response Team training, was used for this series of experiments. The video resolution was $704 \times 576$ at 25 frames per second (fps). The video was encoded using the $\mathrm{x} 265$ open-source high efficiency video coding (HEVC) software, for 23 different rate points (quantization parameters 20-42), and 10 different encoding speed profiles, for a total of 230 video encoding instances.

This work was partly supported by the Marie Curie FP7-PEOPLE-2011IEF DRIVEN project, 301476, and by the National Science Foundation under

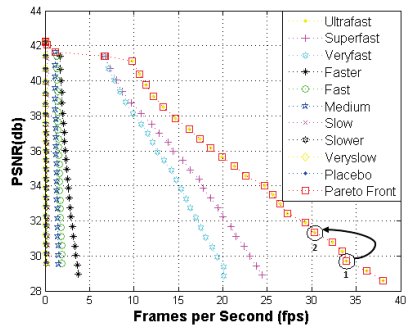

(a) Pareto-front for Video Quality vs Encoding Speed

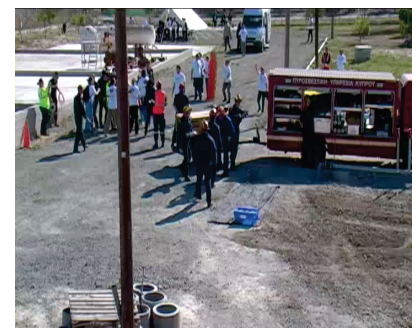

(b) Emergency Scenery Video - 29.7 dB (33.88 fps@226 kbps)
Fig. 1. Adaptive HEVC encoding framework example. In this example, we have adaptation for the maximum video quality mode subject to realtime encoding that translates to frame-rate $\geq 25 \mathrm{fps}$ and available bandwidths of [3]: (1) $\mathrm{BW} \leq 250 \mathrm{kbps}$ to (2) $\mathrm{BW} \leq 384 \mathrm{kbps}$ (max $3 \mathrm{G}$ upload speed). For the lower bandwidth, we achieve a maximum PSNR of 29.7 dB (33.88 fps @ 226 kbps) while the second bandwidth gives a maximum of $31.9 \mathrm{~dB}$ (29.29 fps@363.53 kbps). Encoding delay was 0.426 seconds with 6 core Xeon@2.26 GHz (WPP streams / pool / frames: 18 / 6 $/ 2$ for x.265 encoding).

We show an example of adaptive HEVC encoding to accommodate changing bandwidth in Fig. 1. In this example, we have an increase in available bandwidth from $250 \mathrm{kbps}$ to $384 \mathrm{kbps}$ (3G upload data rates). The video quality improved from $29.7 \mathrm{~dB}$ to $31.9 \mathrm{~dB}$. The emergency video quality was found to be sufficiently good for determining the number of casualties, the total number of people and movements (see Fig. 1(b)). Future work will focus on fast methods for determining the Pareto front, and adaptation among additional models [3].

\section{REFERENCES}

[1] E. Kyriacou, S. Kyprianou, S. Christou, R. Constantinou, A. Panayides, C. Pattichis, "“Avaris Net" Crisis and Emergency Management of Health Services, " 2014 Annual International Conference of the IEEE Engineering in Medicine and Biology Society (EMBC), Aug. 26-30, 2014.

[2] A. S. Panayides, M. S. Pattichis, and C. S. Pattichis, "Mobile-Health Systems Use Diagnostically Driven Medical Video Technologies [Life Sciences]," IEEE Signal Processing Magazine, vol. 30, no. 6, pp. 163172, Nov. 2013.

[3] J. Yuebing, G. Esakki, and M. S. Pattichis, "Dynamically reconfigurable architecture system for time-varying image constraints (DRASTIC) for HEVC intra encoding," Asilomar Conference on Signals, Systems and Computers, 2013, pp.1112-116, Nov. 3-6, 2013. 